\title{
Comparative Analysis of Performance of Routing Protocols in MANETs: A Review
}

\author{
Lakshi Dhiman*, Aman Kumar Sharma** \\ * Department of Computer Science, Himachal Pradesh University \\ *lakshidhiman@gmail.com, **sharmaas1@gmail.com \\ DOI: 10.29322/IJSRP.11.10.2021.p11861 \\ http://dx.doi.org/10.29322/IJSRP.11.10.2021.p11861
}

\begin{abstract}
Routing in MANETs is a difficult challenge because of the mobility of nodes. A number of routing protocols are used to find out the best route. These routing protocols are mainly divided into three types which are Pro-active, Reactive and Hybrid routing protocols. Further, there exists a number of routing protocols which fall under one of these categories.

In this work, four routing protocols, Destination Sequenced Distance Vector (DSDV), Optimized Link State Routing (OLSR), Dynamic Source Routing (DSR) and Ad-hoc On Demand Distance Vector (AODV) are studied and compared after a deep analysis for their performance. It is found that AODV outperforms all other protocols in terms of throughput and packet delivery ratio while OLSR turns out with best performance for end-to-end delay.
\end{abstract}

Index Terms- MANETs, Routing, DSDV, OLSR, DSR, AODV.

\section{INTRODUCTION}

$\mathrm{R}$ outing is a sturdy challenge in Mobile Ad-hoc Networks (MANETs) as it is a wireless network in which nodes are allowed to connect or disconnect with the network at any time without any centralized base station with some prominent features like multi-hoping, elevated flexibility high fault tolerance etc. An exalted characteristic of MANET is that it possesses dynamic topology due to which plenty of chronic and random changes occurs in the topology of MANET which enlarge the difficulty of routing among the mobile nodes of the Mobile Adhoc Network. [2]

Henceforth, AODV (Ad-hoc On-Demand Vector), DSDV (Destination Sequenced Distance Vector), OLSR (Optimized Link State Routing), DSR (Dynamic Source Routing), ZRP (Zone Routing Protocol) and many other ad-hoc routing protocols are designed particularly for MANETs.[20]

\section{OVERVIEW OF ROUTING PROTOCOLS}

Numerous routing protocols and algorithms are put forward for carrying out routing in MANETs. Routing Protocols provides these optimum routes to set up a communication link between a source node and a destination node which are free from selflooping and information redundancy. [1] There are number of routing protocols for MANETs, all of which broadly fall under two main categories which are Proactive Routing Protocols and Reactive Routing protocols. There is an additional type of routing protocols which bunches up the characteristics of both proactive and reactive routing protocols called as Hybrid Routing Protocols. It embark on fully utilizing the minimized network traffic overhead from reactive routing protocols and the reduced communication delays from proactive routing protocols. [3]

This section gives explanation about two proactive routing protocols i.e. DSDV and OLSR followed by two reactive routing protocols which are AODV and DSR.

\section{A. Proactive Routing Protocols}

In proactive or table driven routing protocol every single node uphold one or more routing tables and they are allowed to propagate the information of routing table through the network. Information about the route is available at any instant which reduces the retardation in communication process. These protocols consume more memory and maintenance cost and hence are not relevant in large and extremely dynamic network.

1. Destination Sequenced Distance Vector (DSDV): Destination Sequenced Distance Vector routing protocol was proposed by Charles E. Perkins and Pravin Bhagwat in 1994. The classical Bell-man Ford shortest path routing algorithm is the backbone for the development of the DSDV routing protocol. DSDV was put-forward to brush off the problems like count to infinity, bouncing effect and routing loop problem. [2] In this routing protocol every single node maintains a routing table which incorporates routing information for all other nodes within the Mobile Ad-hoc Network. A routing table in DSDV lists destinations, hop count, next hop and sequence number. [4] Sequence number is a new add-on attribute to the prevailing routing table. Sequence number is coined by the destination node, which contributes in avoiding the loop problems. The route with the most recent sequence number is deemed new. [5] When changes in the network's topology are identified, update messages are disseminated periodically or immediately 
to every single node of the Mobile Ad-hoc Network, [6] using either a broadcast or multicast approach. These update messages are mainly of two types i.e. full dump packets and incremental packets. DSDV assures that the route selected for transmitting a packet is loop free. However, DSDV is not suitable for large networks as it requires more battery power. DSDV cannot be applied in highly dynamic Mobile Ad-hoc Networks as it creates a heavy traffic overhead.

2. Optimized Link State Routing (OLSR): Optimized Link State Routing protocol and has been suggested by Clausen and Jacquet. [2] The primary reason for the development of OLSR routing protocol is to disseminate topology information in such a manner that the use of flooding technique is kept down. [1] In OLSR, ever node of the Mobile Ad-hoc Network retains the statistics about network topology by regularly exchanging the link state messages. OLSR adopts an effective technology for forwarding the link state messages, called as Multipoint Relaying (MPR) [2] in order to cut down the network overhead and to reduce flooding. MPRs rare some selected nodes that are permitted to broadcast the messages in the network. When compared to a pure flooding system, this strategy of using the MPRs significantly reduces message overhead. In OLSR protocol, routing process is carried out via various control messages which are Hello Message, Topology Control Message, MID (Multiple Interface Declaration) message and HNA (Host and Network) Message. Hello Messages are used to obtain the information about a host's neighbor which helps in framing the MPR selector set. Topology Control Messages are aimed at calculating the route and only MPR nodes are allowed to broadcast the topology control messages over the entire network. [7] MID message declares the presence of multiple interfaces on a node and provides a list of IP addresses of all the nodes for broadcasting these messages on more than one interface. HNA Message is used to connect the OLSR network to other networks via a gateway node that announces OLSR network routing information to other networks. [8] The OLSR protocol do not allow for extended delays in data packet transmission. The OLSR framework is intended for dense networks in which communication between a large numbers of nodes is expected to occur regularly.

B. Reactive Routing Protocols: In reactive routing protocols, nodes do not uphold any routing information when it is not required. These protocols instantly hunt a route when it is on-demand, therefore also called as on-demand routing protocols. These protocols cut down the network traffic overhead and memory usage as compared to proactive routing protocols.

1. Dynamic Source Routing (DSR): Dynamic Source Routing (DSR) protocol was originally drafted by D.B Johnson, Maltz and Broch. This protocol does not rely upon periodic updates and hence eradicates the core requirement of pro-active routing protocols by wiping out the usage of periodic table updates in Ad-hoc wireless networks, which in result diminish the bandwidth consumed by control packets. A marking trait of Dynamic source routing protocol is the usage of source routing. Source routing is a technique by virtue of which a sender node identifies the whole sequence of multiple hops through which the packet must traverse to reach the receiver node. [9] Every node has a route cache which contains the information about the present routes and this route cache is refreshed when new routes are found. When a node has data packets to send, it instantly searches the routing table in the cache to see if a route to the required sink exists. If the sender node discovers numerous routes, one with the minimum hop count is chosen and included as a header of packet which is being sent. [10] DSR protocols imparts outstanding performance in multi-hop MANETs as it sinks the routing overhead to the minimum. DSR is made up of two basic mechanisms: "Route Discovery" and "Route Maintenance":

Route Discovery: Route discovery focuses on discovering routes from a sender node to a receiver node within a mobile ad-hoc network with the help of RREQ and RREP message along with a route cache.

Route maintenance: The process of route maintenance is executed when an already existing path fails and a RERR message is received.

2. Ad-Hoc On-Demand Distance Vector (AODV): Ad-hoc On-Demand Distance Vector (AODV) Routing Protocol is a result of unification of DSR and DSDV routing protocols which was given by C.E Perkins and E.M Royer. Elemental mechanisms of route discovery and route maintenance are inherited from DSR protocol and use of sequence numbers is taken from DSDV. In AODV, maintenance of routes is time-based which means routes are kept for as long as the source requires them.[11] Packets in AODV simply provide the destination address without holding 
any information about the complete path.[12] Routing starts with broadcasting a route request(RREQ) packet from a source to adjoining nodes. These nodes further broadcast the RREQ packet till it reaches the route information for destination or destination itself. RREQ packet contains source ID, Destination ID, recent sequence number, hop count, broadcast ID, and time to live (TTL). TTL is a small value commenced initially with RREQ packet which is incremented until it reaches a definite threshold value. Each time a route is utilized its lifetime is

\section{RESULTS AND ANALYSIS}

In this research article, routing protocols DSDV, OLSR, DSR and AODV are compared by employing a theoretical approach for detailed examination of performance and behavior of these protocols when they are analyzed for a number of different simulation environments:

1. A simulation was performed for a Mobile Ad-hoc Network with 12 nodes for a time period of 150 seconds using NS 2.35. In this experiment two separate TCP connections conveying FTP Traffic were utilized and analyzed independently for different routing protocols such as AODV, DSDV and DSR. Their performance is evaluated by changing the number of nodes for both TCP connections. After simulation, it is observed that for a highly frequent network, performance of AODV is better and for a less mobile network DSR performs well. [13]

2. A simulation environment was devised in which nodes of MANET may move about freely and communicate while doing so. Two distinct scenarios were utilized in an IPv6 environment with a wireless LAN server to assess the performance of different routing protocols using the protocol's default settings. On the basis of three performance metrics which are load, delay and throughput it is concluded that routing protocols AODV and DSR have outperformed all other protocols. [14]

3. Using the NS-2 network simulator, to compare ad-hoc routing protocols in MANETs using a variety of analytical network performance measures, a simulation was performed. In an area of $800 \mathrm{~m} * 800 \mathrm{~m}$, the network considered was comprised of 6 nodes with constant bit rate (CBR) traffic with data packets of 512 byte packet size. The results of the simulation for primary parameters actually reveal that AODV routing protocol performs best in average throughput and can be improved for average packet delivery ratio. [15]

4. A research employs a simulation to assess the performance of DSDV, OLSR, AODV, and DSR routing protocols in both CBR (Constant Bit Rate) and VBR reset which is calculated by adding the current time and an active route timeout parameter.

If a node is the destination or has a valid route to the destination, it returns an optimal route to the source with a route reply message (RREP) by unicasting it which contains source address, destination address, destination sequence number, hop count and life time. A route error (RERR) message is created when a node in an active route is lost, informing the remaining nodes on both sides of the link about the loss. [11]

(Variable Bit Rate) traffic by changing node density, transmission rate and transmission range. To investigate the functioning of protocols, a simulation environment was constructed using NS-2.35 simulator on an Ubuntu 14.04 platform. Performance was analyzed with different number of nodes ranging from 20 to 100 in an area of $1000 \mathrm{~m} * 1000 \mathrm{~m}$ for simulation time of 300 seconds. The evaluation is carried out by transmitting packets of size 1024 bytes at a node speed of $10 \mathrm{~m} / \mathrm{s}$ for a pause duration of 10 seconds. The results of the simulation performed determined that AODV is best routing protocol when packet delivery ratio, end-to-end delay, throughput and jitter metrics are considered. [16]

5. A research work was carried out to evaluate the performance of routing protocols for CBR (Constant Bit Rate) and FTP traffic, by altering the number of nodes in terms of throughput, end-to-end delay and packet loss. The network simulator (NS-2.35) was used to run the simulation with Linux operating system. Simulation was accomplished for two simulation durations of 10 seconds and 20 seconds in a simulation area of $1000 \mathrm{~m} * 1000 \mathrm{~m}$. Mobility of nodes was kept random with varying the number of nodes as 5, 8, 10, 15 and 20. There were two sizes of packets used: 512 bytes and 1024 bytes with deviating data rates. Results of the simulation shows that Every protocol worked well at the peer level, but when they are evaluated for efficiency using the tool NS2 and TCL scripts, the simulation results show that AODV has a greater throughput and OLSR has a superior performance in both packet loss rate and latency.[17]

6. A research article tested the performance of different routing protocols for Mobile Ad-Hoc networks. Using the NS-2 programme for simulation, the research results and network assessment are made, based on network size, mobility and changeable network load. On a $500 \mathrm{~m}^{2}$ simulation area, 50 nodes were used in the network setup. For 100 seconds, the simulation was run and the packet size was set to 512 bytes. Each protocol has a transmit buffer of 64 packs. The output was generated for AWK 
scripts with analogous simulation settings, in which the node velocity was set to $20 \mathrm{~m} / \mathrm{s}$ and the waiting period was varied between $0,10,20,40$, and 100 seconds. The AODV and DSR routing protocols performed exceptionally well in simulations, delivering around 85 percent of the data packets, and another conclusion derived was that the AODV and DSR protocols performed better in fast mobility simulations than the DSDV protocol. [18]

7. A simulation environment was set up to bring off a simulation on Network Simulator-2 (NS-2) for different mobility speed of nodes which are $0 \mathrm{~m} / \mathrm{s}, 1 \mathrm{~m} / \mathrm{s}, 5 \mathrm{~m} / \mathrm{s}$ and $10 \mathrm{~m} / \mathrm{s}$ to evaluate the performance of AODV, DSDV, OLSR and DSR routing protocols quantitatively for an ad-hoc network with 30,50 and 80 nodes. For each routing protocol, the results are summarized as a function of the number of nodes and their mobilities for throughput, end-to-end delay and packet delivery ratio which shows that overall, the AODV and DSR protocols outperforms the DSDV and OLSR protocols and are best options for wider or bigger networks.[19]

8. For assessing the efficiency of the MANET routing protocols a simulation was carried out on network

\section{CONCLUSION AND FUTURE SCOPE}

Mobile Ad-hoc Networks are gaining attraction of researchers as MANETs can be set up anywhere and anytime without any fixed infrastructure. Routing is a challenging task in MANETs due to the dynamic nature of nodes. The crucial challenge for mobile adhoc networks is to route packets with maximum throughput and least end-to-end delay even when the conditions are highly dynamic.

In this study, various routing protocols are compared by reviewing the literature which concludes that overall performance of AODV is better than others even for high number of nodes with faster mobility speed. After AODV, DSR also functions well in terms of throughput and packet delivery ratio. Results obtained from OLSR are excellent for end-to end delay whereas DSDV does not give efficient results in any case. It can be concluded that AODV is best reactive routing protocol while on the other hand for proactive routing protocols OLSR is performs better. Performance and efficiency of AODV protocol can be enhanced in future so that it can provide higher security while transmitting the packets.

\section{REFERENCES}

[1] Kritika Lamba, Aprajita Rawat, Shelja Sharma and Dr. Prateek Jain, "An Analysis Based on Comparative Study of Routing Protocols in MANETs" International Journal of Engineering Research and Technology (IJERT), Vol 7. Issue-09, September-2018.

[2] Anuj K. Gupta, Harsh Sadawarti, and Anil K. Verma, "Review of Various Routing Protocols for MANETs" International Journal of Information and Electronics Engineering, Vol. 1, No. 3, November-2011 simulator 3.25 with IEEE 802.11 MAC protocol when traffic sources taken are CBR (Constant Bit Rate) traffic. Random waypoint mobility model was used for simulation with a network size of $500 \mathrm{~m} * 2000 \mathrm{~m}$ for 20 , 60 and 100 mobile nodes. Performance of routing protocols is investigated by transmitting a packet of size 64 byte with 4 packets per second rate of transmission for 200 seconds simulation time with a constant speed of $20 \mathrm{~m} / \mathrm{s}$ for $0,10,30,50,70$ and 100 seconds pause time. Routing protocols were compared depending on the function of pause time and number of sources used which set forth that the best overall performance was achieved by AODV, which is appropriate for networks with a larger number of nodes and DSR, on the other hand, is best suited to networks with limited mobility and low traffic density.[20]

According to the results of these experiments and simulations, the AODV routing protocol outperforms all other routing protocols in different network configurations; nevertheless the performance of DSR and OLSR routing protocols is good in some cases after AODV

[3] Alex Hinds, Michael Ngulube, Shaoying Zhu, and Hussain Al-Aqrabi, "A Review of Routing Protocols for Mobile Ad-Hoc NETworks (MANET)" International Journal of Information and Education Technology, Vol. 3, No. 1, February 2013

[4] Guoyou He, "Destination-Sequenced Distance Vector (DSDV) Protocol"

[5] Parul Gupta, "A Literature Survey of MANET" International Research Journal of Engineering and Technology (IRJET), Vol. 3 Issue2, February-2016

[6] Mr. Uday K. Patil and Dr. Mrs. S.R. Chougule, "MANET-Evaluation of DSDV and AROD Routing Protocol" International Journal of Engineering Sciences \& Research Technology, ISSN: 2277-9655, March2014

[7] Janak kumar Patel and Mitesh Thakkar, "OLSR Protocol with Cross Layer Design in MANET" International journal of Engineering development and research (IJDER), Vol.2 Issue-2, 2014

[8] Mubarak Mohammed Al-Ezzi Sufyan and Khalid Saeed, "Performance Evaluation of OLSR Routing Protocol using Different Mobility Models in MANETS" International Journal of Computer Applications (0975 - 8887),Vol. 137 No.8, March-2016

[9] Prabhleen Kaur and Sukhman, "An Overview on MANETAdvantages, Characteristics and Security Attacks" International Journal of Computer Applications (0975 - 8887), 4th International Conference on Advancements in Engineering \& Technology (ICAET 2016)

[10] Salem A. Almazok and Bülent Bilgehan, "A novel dynamic source routing (DSR) protocol based on minimum execution time scheduling and moth flame optimization (MET-MFO)" EURASIP Journal on Wireless Communications and Networking, Vol. 2020, Issue 1,December-2020

[11] Prashant Kumar Maurya, Gaurav Sharma, Vaishali Sahu, Ashish Roberts and Mahendra Srivastava, "An Overview of AODV Routing 
Protocol" International Journal of Modern Engineering Research (IJMER), Vol.2 Issue.3, May-June 2012

[12] Ms. Sangeeta Singh, Dr. Pardeep Kumar and Mrs. Shalini Aggarwal," Performance Metric Comparison of AODV and DSR Routing Protocols in MANETS" International Journal for Scientific Research \& Development (IJSRD) Vol. 3 Issue 04, 2015

[13] Kalpesh A. Popat, Dr. Priyanka Sharma and Hardik Molia, "A Study of Routing Protocols for MANETs" Proceedings of the International Congress on Information and Communication Technology, January-2016 [14] Astha Mishra, Shweta Singh and Arun Kumar Tripathi, "Comparison of MANET Routing Protocols" International Journal of Computer Science and Mobile Computing (IJCSMC), Vol. 8 Issue- 2, February2019

[15] Ashutosh Sharma and Rajiv Kumar, "Performance Comparison and Detailed Study of AODV, DSDV, DSR, TORA and OLSR Routing Protocols in Ad-Hoc Networks" 2016 Fourth International Conference on Parallel Distributed and Grid Computing (PGDC)

[16] K. Gangadhara Rao, Ch. Suresh Babu , B. Basaveswara Rao and D. Venkatesulu, "Simulation Based Performance Evaluation of Various Routing Protocols in MANETs" IOSR Journal of Mobile Computing \& Application (IOSR-JMCA), e-ISSN-2394-0050, P-ISSN- 23940042. Volume 3, Issue 4 (Jul. - Aug. 2016), PP 23-39

[17] Katroth Balakrishna Maruthiram and Kare Suresh Babu, "Performance Comparison of DSDV, OLSR, DSR and AODV MANET
Routing Protocols in Traffic Conditions" International Journal of Science and Research (IJSR) ISSN (Online)- 2319-7064

[18] Murad Ghazy Khalaf Alabdullah, Bassam Mohsin Atiyah, Kaesar Sabah Khalaf and Saber Hameed Yadgar, "Analysis and simulation of three MANET routing protocols: A research on AODV, DSR \& DSDV characteristics and their performance evaluation" Periodicals of Engineering and Natural Sciences ISSN 2303-4521, Vol.7, No.3, September 2019, pp.1228-1238

[19] Lavanya Poluboyina , V S K Reddy and A Mallikarjuna Prasad, "Performance Comparison of DSDV, OLSR, AODV and DSR for Mobile Ad hoc Networks" International Journal of Emerging Technology and Advanced Engineering (IJETAE), Vol. 8 Issue 1, January -2018

[20] Akshay Shankar and Lavanya Chelle, "Performance Comparison of AODV, DSR, DSDV and OLSR MANET Routing Protocols" International Journal of Engineering Research \& Technology (IJERT), Vol. 5 Issue 10, October-2016

\section{AUTHORS}

First Author - Lakshi Dhiman, M.Tech (CS), Himachal Pradesh University and lakshidhiman@gmail.com.

Second Author - Aman Kumar Sharma, Ph.D., Professor at Himachal Pradesh University and sharmaas1@gmail.com. 